\title{
Electromagnetic effects on turbulent transport in high-performance ASDEX Upgrade discharges
}

\author{
H. Doerk, ${ }^{1}$ M. Dunne, ${ }^{1}$ F. Jenko, ${ }^{1,2}$ F. Ryter,${ }^{1}$ \\ P. A. Schneider, ${ }^{1}$ E. Wolfrum, ${ }^{1}$ and the ASDEX Upgrade Team ${ }^{1}$ \\ ${ }^{1}$ Max-Planck-Institut für Plasmaphysik, Boltzmannstraße 2, D-85748 Garching, Germany \\ ${ }^{2}$ University of California, Los Angeles, California 90095, USA
}

\begin{abstract}
Modern tokamak H-mode discharges routinely operate at high plasma beta. Dedicated experiments performed on multiple machines measure contradicting dependence of the plasma confinement on this important parameter. In view of designing high-performance scenarios for next-generation devices like ITER, a fundamental understanding of the involved physics is crucial. Theoretical results - most of which have been obtained for simplified setups - indicate that increased beta does not only modify the characteristics of microturbulence, but also potentially introduces fundamentally new physics. Empowered by highly accurate measurements at ASDEX Upgrade, the GENE turbulence code is used to perform a comprehensive gyrokinetic study of dedicated H-Mode plasmas. We find the stabilization of ion-temperature-gradient driven turbulence to be the most pronounced beta effect in these experimentally relevant cases. The resulting beta-improved core confinement should thus be considered for extrapolations to future machines.
\end{abstract}

\section{INTRODUCTION}

Modern tokamak experiments routinely reach high plasma confinement in H-mode operation [1]. In view of designing high-performance fusion plasmas, the beta $(\beta)$ parameter - the ratio of kinetic to magnetic pressure - is desired to be large (up to certain macroscopic stability constraints). This is due to the strong $\left(\sim \beta^{2}\right)$ increase of both fusion gain and bootstrap fraction with beta. Experiments on the $\beta$-scaling of plasma confinement have been performed in many machines, [2-6], which are reviewed in the context of dimensionless parameter analysis [7, 8]. Different, even contradicting trends are found, indicating that the widely used ITER physics base scaling IPB98 $(y, 2)[9]$ may not universally apply.

This paper addresses the impact of $\beta$ on microturbulence, which is a key limiting factor for energy and particle confinement. Several turbulencedriving microinstabilities are known. Some of them exist in the electrostatic limit $(\beta \rightarrow 0)$, because they primarily involve electric field fluctuations. Others involve magnetic fluctuations and thus (through Ampère's law) require finite $\beta$. Prominent electrostatic instabilities are the ion temperature gradient (ITG) driven instability, its electron-scale analogue, the electron temperature gradient (ETG) driven instability and the trapped electron mode (TEM).

In terms of electromagnetic modifications, there is evidence for a $\beta$-induced reduction of ETG transport under edge conditions, even though linear physics remains unmodified [10]. Also TEMs are hardly affected by $\beta$ in terms of linear physics $[11,12]$. Nonlinearly, a slight TEM transport increase is found for certain core parameters [13]. For the ITG mode, however, analytical considerations show a successive stabilization with increasing $\beta$ until a transition into the kinetic ballooning mode (KBM) takes place above a certain critical value

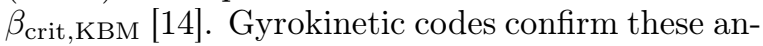
alytical results [11-13, 15] and furthermore demonstrate that ITG stabilization not only carries over to the turbulent regime, but that nonlinear physics can even lead to an up-shift of the critical temperature gradient [13]. Nonlinear simulations in the $\mathrm{KBM}$ regime have been attempted $[12,16]$ indicating that the threshold level for the onset of KBM transport roughly coincides with the linear one.

Besides KBM turbulence, microtearing mode (MTM) turbulence exhibits a critical $\beta$ value as well. The MTM instability mechanism is the formation of small-scale magnetic islands driven by a current, which is reinforced by the electron temperature gradient. Although MTMs have been discussed since decades [17-21] nonlinear gyrokinetic results confirmed their potential importance for electron heat transport only a few years ago [22-24]. Several facets of linear MTM physics have been studied [25-29] and there is evidence for a role of MTMs in spherical tokamak pedestal formation $[30,31]$ or at the top of the pedestal in standard tokamaks $[32,33]$.

A third critical $\beta$ threshold has been revealed in strongly driven ITG turbulence. Above this socalled non-zonal transition (NZT), zonal flows are short-circuited by magnetic fluctuations, leading to high transport levels $[34,35]$. Even if none of the mentioned critical beta thresholds (KBM, MTM or NZT) is overcome, magnetic transport may become significant in ITG turbulence at larger beta $[10-13,36,37]$ since it scales as $\beta^{2}$. This has been traced back to the nonlinear excitation of subdominant microtearing modes [38, 39].

However, as many of the above findings are obtained in simplified setups, the obvious question is, 
which of them are relevant for concrete experimental cases and thus also for future machines. This paper contributes by merging advances from experimental and theoretical research by performing first-principle nonlinear gyrokinetic computations for selected ASDEX Upgrade discharges.

Recent developments at ASDEX Upgrade allow to measure the ion and electron kinetic profiles with greatly improved precision. At the same time, gyrokinetic codes have been further developed to include comprehensive physics, such as realistic geometry, parallel and perpendicular magnetic fluctuations, background flow shear and sophisticated collision operators.

Comparisons of macroscopic quantities, like transport coefficients, and microscopic turbulence characteristics, such as fluctuation amplitudes and cross-phases have already been demonstrated for low beta plasmas [40-42]. With respect to electromagnetic effects, the transition to experimentally relevant setups has recently been started for several JET cases, where nonlinear $\beta$-stabilization of ITG turbulence is confirmed and a strong contribution of fast ions is identified [43, 44].

In this paper, four ASDEX Upgrade H-modes (AUG\#29197, AUG\#29224, AUG\#26459 and AUG\#23227) are investigated. The first two are part of a dedicated beta variation. Attempting the highest available degree of realism, a comprehensive gyrokinetic study is performed using the GENE turbulence code [45]. In Sec. II details on the numerical and experimental setup are provided, in Sec. III an analysis of microinstabilities is presented and in Sec. IV nonlinear physics is discussed and turbulent fluxes are compared to experimental results. Conclusions are drawn in Sec. V. The overall objective is to identify key physics effects that are necessary to understand turbulent heat transport in these experimental cases, thereby allowing for more reliable predictions for future fusion devices.

\section{PHYSICAL AND NUMERICAL SETUP}

\section{A. Numerical setup and the GENE code}

The gyrokinetic turbulence code GENE [45] is used for the simulation work presented in the following. GENE solves the gyrokinetic Vlasov equation on a fixed grid in 5-dimensional $\left\{x, y, z, v_{\|}, \mu\right\}$ phase space and allows for an arbitrary number of plasma species. A comprehensive LandauBoltzmann collision operator is included [46, 47]. Although radial (or binormal) background profile variations can be considered in the global code version [48], we apply the local flux-tube model throughout this work, which is justified by the smallness of the ion gyroradius compared to the machine size.

Two types of electromagnetic effects should be distinguished: Dynamical effects due to perpendicular and parallel magnetic field fluctuations and geometric effects originating from the fact that $\beta^{\prime}$ is proportional the pressure gradient, which is essential for the magnetic equilibrium. Plasma geometry and magnetic drifts should thus be kept consistent with $\beta$ and its gradient. To that aim, interfaces to several MHD equilibrium codes allow the use of realistic plasma shapes. Also analytical geometry, such as the Miller-, circular-, and s-alpha model are available.

For nonlinear simulations, the domain size is about $l_{x} \times l_{y} \approx 100 \rho_{s} \times 120 \rho_{s}$ in the perpendicular spatial directions. Here, $\rho_{s}=c_{s} / \Omega_{i}$ is the ion gyroradius at electron temperature $T_{0 e}, c_{s}=$ $\left(T_{0 e} / m_{i}\right)^{1 / 2}$ is the sound speed, $\Omega_{i}=m_{i} c /\left(e B_{0}\right)$ is the Larmor frequency, $B_{0}$ is the guiding field strength at the magnetic axis and $m_{j}$ is the particle mass of species $j$. Along the field line the domains size is $l_{z}=2 \pi$ and $l_{v} \times l_{\mu}=3 v_{T} \times 9 T_{0} / B_{0}$ is typically chosen for the velocity space domain. Here, $v_{T}=\left(2 T_{0} / m\right)^{1 / 2}$ is the thermal velocity of the corresponding plasma species. The nominal number of grid points is $n_{x} \times n_{y} \times n_{z} \times n_{v} \times n_{\mu}=$ $384 \times 96 \times 32 \times 32 \times 16$.

Although rigorous convergence tests (e.g. doubling resolution in all dimensions) become prohibitive in terms of computational cost, the performed tests lead to the conclusion that the relevant physics effects are sufficiently resolved. Since MTMs are known to exist on small radial scales, a special focus is put on this dimension. The gyrokinetic large eddy simulation technique (GyroLES) [49] is used to model the transfer of free energy to smaller scales in the $x, y$ plane. This is done by adding $k_{x}$ and $k_{y}$ hyperdiffusion terms, with species-dependent coefficients calibrated dynamically during the simulation. It has been verified on a few of our cases that at nominal resolution virtually no changes of the overall fluctuation levels and fluxes occur, while the high- $k$ part of the turbulent spectra shows a more clear power law behavior, as expected. Linear simulations determine the growth rate for a single wavenumber $k_{y}$ and use $n_{x} \approx 25$, while $l_{x}$ is adapted to $k_{y}$ according to the quasi-periodic parallel boundary condition of the flux-tube model [50].

\section{B. Definition of plasma parameters}

Throughout this paper, the following definitions of plasma parameters are applied. As a radial coordinate, we chose $\rho_{\text {tor }}$, the square root of the toroidal magnetic flux $\Phi$, normalized to its value 
$\Phi_{\text {sep }}$ at the separatrix. The macroscopic reference length is defined as $\alpha \equiv\left(\Phi_{\mathrm{sep}} / \pi B_{0}\right)^{1 / 2}$. For this study, the most relevant global discharge parameters is $\beta_{N}=\beta_{t h}[\%] /\left(I_{p}[\mathrm{MA}] / a[\mathrm{~m}] B_{0}[\mathrm{~T}]\right)$, where $I_{p}$ is the total plasma current, $a$ is the tokamak minor radius and $\beta_{\text {th }}$ is the volume averaged thermal plasma beta.

Local parameters are obtained by evaluating experimental data (such as density $n_{0 j}$ and temperature $T_{0 j}$ ) at a reference radial position $\rho_{\text {tor }}=x_{0}$. Geometric parameters are the safety factor $q$ and the magnetic shear $\hat{s}=\left(\rho_{\text {tor }} / q\right) \mathrm{d} q / \mathrm{d} \rho_{\text {tor }}$ and $\epsilon=$ $x_{0} /(R / \alpha)$, with $R$ the tokamak major radius. The local electron beta is defined as $\beta_{e}=8 \pi n_{0 e} T_{0 e} / B_{0}^{2}$ and $\rho^{\star}=\rho_{s} / \alpha$. The collision rate for species $j$ of charge $q_{j}$ colliding with species $j^{\prime}$ is defined as $\nu_{j j^{\prime}}=4 \pi n_{0 j} q_{j}^{2} q_{j^{\prime}}^{2} \ln \Lambda\left(2 T_{0 j}\right)^{-3 / 2} m_{j}^{-1 / 2}$, with $\ln \Lambda$ being the Coulomb logarithm that is approximated to be species-independent. Normalized to the ion bounce frequency, the dimensionless ion collision rate becomes $\nu_{i}^{\star}=4 \nu_{i i} R_{0} q /\left(3 \sqrt{\pi} v_{T i} \epsilon^{3 / 2}\right)$.

Normalized logarithmic temperature gradients are defined as $\alpha / L_{T j}=-\left(1 / T_{0 j}\right) \mathrm{d} T_{0 j} / \mathrm{d} \rho_{\text {tor }}$, and $\alpha / L_{n j}$ is defined accordingly. Moreover, the effect of a sheared toroidal plasma rotation $\Omega_{\text {tor }}$ can be considered. The normalized rate of equilibrium flow shear is $\hat{\gamma}_{E}=\gamma_{E} /\left(c_{s} / \alpha\right)=-x_{0} / q \Omega_{\text {tor }}^{\prime}$, with $\Omega_{\text {tor }}^{\prime}$ being the derivative of the rotation profile with respect to $\rho_{\text {tor }}$. The parallel component of $\Omega_{\text {tor }}$ results in a parallel flow shearing rate $\gamma_{\mathrm{PFS}}$, which contributes to the driving terms. In our convention, the normalized magnitude is consistent when $\hat{\gamma}_{P F S}=\hat{\gamma}_{E}$ is set.

\section{Experimental parameters}

Experimental measurements from four H-Mode discharges performed in ASDEX Upgrade form the basis of the present gyrokinetic study. The equilibrium information is taken from the experimental equilibrium calculated by the CLISTE code including kinetic data, [51]. The heat fluxes are provided by power balance analysis with the transport TRANSP code, [52]. Global discharge parameters are summarized in Table I. By selecting reference positions we define five nominal parameter sets listed in Table II.

The main focus of this work is put on analyzing the pair of ASDEX Upgrade H-Mode discharges 29197 and 29224, which have been designed to solely vary $\beta$ while keeping $\nu^{*}$ and $\rho^{*}$ constant. For this purpose the usual method has been used, in which $B_{0}$ is varied and the controlled parameter, plasma current $\left(I_{p}\right)$, heating power $(P)$ and density $(n)$ are adjusted according to: $I_{p} \sim B_{0}$, $n \sim B_{0}^{4}$ with $\beta \sim B_{0}^{4}$. The heating power is expected to vary as $P \sim B_{0}^{4}$ from gyro-Bohm scaling, while the temperature follows $T \sim B_{0}^{2}$.

Fulfilling this over the whole radius, is a rather complex experimental goal and the experiments used for the present study were by far not perfect. There was a mismatch of the heat deposition profiles and the temperature profile measurements have large uncertainties. The density profiles of the high and low beta cases also do not match well. However, in the present pair of discharges, $\beta$ varies by a factor of two, and the remaining dimensionless parameters are sufficiently similar for the purpose of discussing the main physics results yielded by our gyrokinetic calculations. The quality of the global confinement is measured in terms of the normalized thermal energy confinement time $\tau_{E} \Omega_{i}$, for which we have $\tau_{E} \Omega_{i} \sim \beta_{N}^{-0.18}$ when assuming a power-law behavior between those two operation points. Thus, the beta degradation appears to be weaker than in previous ASDEX Upgrade measurements, [5]. Knowing parameter dependencies of $\tau_{E}$ is important for the design of operation scenarios in existing and future machines.

We select the radial position $\rho_{\text {tor }}=0.5$, defining case A from AUG $\# 29197$ at time $t=1.72 \mathrm{~s}($ low $\beta$ ) and case B from AUG\#29224 at $t=4.72 \mathrm{~s}$ (high $\beta$ ). Measured temperature profiles are depicted in Fig. 1. Note that the $T_{e}$ profile for AUG $\# 29197$ exhibits a radial oscillation which is attributed to systematic uncertainties in the calibration. However $T_{e}$ remains close to $T_{i}$, such that, considering the relatively high plasma density, it is reasonable to set $T_{e}=T_{i}$. The $\alpha / L_{T}$ values of Table II are obtained from local fits to the nominal profiles. The uncertainties on the gradients are rather large (see also Section IV B). More robust is the observation that the core temperature is much higher in the high beta discharge, while the edge temperatures are similar. The trend of a larger (logarithmic) gradient at higher beta is also illustrated in Fig. 1 for the ion data. Since gyrokinetic results tend to be very sensitive to the temperature gradients, variations in $\alpha / L_{T}=\alpha / L_{T i}=\alpha / L_{T e}$ are performed in the following sections.

To supplement cases A and B, we use three additional parameter sets from the following experimental cases. Case $\mathrm{C}$ is extracted from case B discharge AUG $\# 29224$, also taken at $t=4.72 \mathrm{~s}$, but radially further out, at $\rho_{\text {tor }}=0.75$. The values of $\alpha / L_{T}$ are reduced by about $50 \%$ with respect to fits to the experimental profile. A more accurate comparison between simulation and experiment at this more outward radial position is scheduled for future work.

Case D corresponds to AUG\#26459 at $t=4.0 \mathrm{~s}$ and $\rho_{\text {tor }}=0.65$, for which linear results have already been published in Ref. [27] (here, we use a refined fit to the temperature and density profiles). The configuration of this discharge is close 

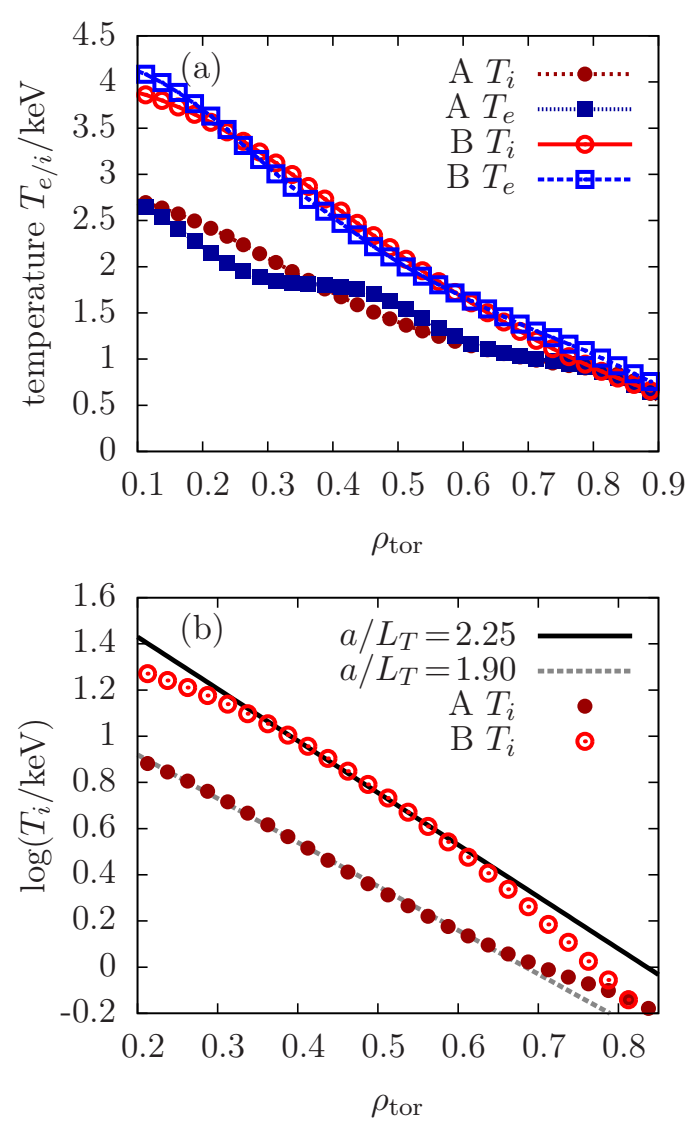

Figure 1: (a) Experimentally determined temperature profiles for cases A and B. The logarithmic plot (b) indicates that $\alpha / L_{T i}$ is increased in the high beta case, especially in the outer core.

to double-null and shows Type-II ELMs, supported by relatively high density and high triangularity $[53,54]$. However, at the selected radial position, the parameters are not far from usual H-mode operation points.

Finally, case E parameters are taken from the improved H-Mode discharge AUG\#23227 at $t=$ $5.1 \mathrm{~s}$ and $\rho_{\text {tor }}=0.75$. Improved H-modes (or hybrid scenarios) are designed to yield high confinement at low plasma current, which is beneficial for long pulses and plasma stability [55].

Although not all of these selected discharges pursue the same physics goals, the general focus is put on generating high-performance, i.e. reaching a high confinement time. In particular cases B and E use comparably high heating power to achieve this.

\begin{tabular}{|c|c|c|c|c|}
\hline AUG $\#$ & 29197 & 29224 & 26459 & 23227 \\
\hline \hline$\beta_{\mathrm{N}, \text { th }}$ & 1.67 & 2.6 & 1.94 & 1.83 \\
\hline$I_{\mathrm{p}} / \mathrm{MA}$ & 0.938 & 1.05 & 1.00 & 1.01 \\
\hline$\alpha / \mathrm{m}$ & 0.645 & 0.645 & 0.646 & 0.643 \\
\hline$\left|B_{0}\right| / \mathrm{T}$ & 2.17 & 2.37 & 2.37 & 2.44 \\
\hline$\tau_{\mathrm{E}, \mathrm{th}} / \mathrm{s}$ & 0.071 & 0.06 & 0.066 & 0.094 \\
\hline$\tau_{\mathrm{E}, \mathrm{th}} B_{0}$ & 0.154 & 0.142 & 0.156 & 0.22 \\
\hline
\end{tabular}

Table I: Global plasma parameters for the four selected AUG discharges.

\begin{tabular}{|c|c|c|c|c|c|}
\hline case & $\mathrm{A}$ & $\mathrm{B}$ & $\mathrm{C}$ & $\mathrm{D}$ & $\mathrm{E}$ \\
\hline $\mathrm{AUG} \#$ & 29197 & 29224 & 29224 & 26459 & 23227 \\
\hline \hline$\rho_{\text {tor }}$ & 0.5 & 0.5 & 0.75 & 0.65 & 0.7 \\
\hline$\nu_{i}^{\star}$ & 0.0756 & 0.0580 & 0.143 & 0.294 & 0.0463 \\
\hline $1 / \rho^{\star}$ & 252 & 233 & 299 & 381 & 291 \\
\hline$\beta_{e}[\%]$ & 0.815 & 1.55 & 0.882 & 0.40 & 0.60 \\
\hline$T_{i} / T_{e}$ & 1 & 1 & 1 & 1 & 1.31 \\
\hline$q$ & 1.48 & 1.34 & 2.48 & 2.45 & 2.35 \\
\hline$\hat{s}$ & 1.31 & 1.08 & 2.10 & 1.58 & 1.94 \\
\hline$R / \alpha$ & 2.62 & 2.63 & 2.63 & 2.67 & 2.67 \\
\hline$\alpha / L_{T e}$ & 1.9 & 2.25 & 2.0 & 3.0 & 1.73 \\
\hline$\alpha / L_{T i}$ & 1.9 & 2.25 & 2.0 & 2.0 & 2.61 \\
\hline$\alpha / L_{n e}$ & 0.23 & 0.1 & 0.3 & 0.27 & 0.411 \\
\hline$\hat{\gamma}_{E \times B}$ & 0.046 & 0.056 & 0.073 & 0.05 & 0.068 \\
\hline$Z_{\text {eff }}$ & 1.4 & 1.4 & 1.4 & 1 & 1.5 \\
\hline$T_{0 e} / \mathrm{keV}$ & 1.48 & 2.05 & 1.25 & 0.672 & 1.39 \\
\hline$n_{0 e}\left[10^{19} / \mathrm{m}^{3}\right]$ & 6.42 & 10.5 & 9.80 & 7.03 & 6.36 \\
\hline
\end{tabular}

Table II: Nominal local parameters for the five considered cases.

\section{MICROINSTABILITY ANALYSIS}

\section{A. Wavenumber spectra}

In this Section, a linear gyrokinetic stability analysis is performed for all cases from Tab. II. Growth rates $\gamma$ and frequencies $\omega$ of the most unstable solutions are depicted in Fig. 2 as a function of wavenumber $k_{y}$. Dominant $\alpha / L_{T i}$-drive, positive (ion-diamagnetic) frequency, and ballooning parity identify ITG modes. MTMs are recognized by tearing parity, strong $\alpha / L_{T e}$-drive, negative frequency, and dominant magnetic transport. A common feature is the robust co-existence of ITG and MTM, whereby MTMs tend to be unstable at lower $k_{y}$ and smaller $\gamma$. In case B, wavenumber ranges of ITG and MTM substantially overlap and growth rates are of similar magnitude.

By performing scans in $\alpha / L_{n}$ and $\alpha / L_{T}$ around the nominal values, it is verified that the MTM is stabilized by increased $\alpha / L_{n}$ and de-stabilized by $\alpha / L_{T e}$. The ITG mode is slightly destabilized 

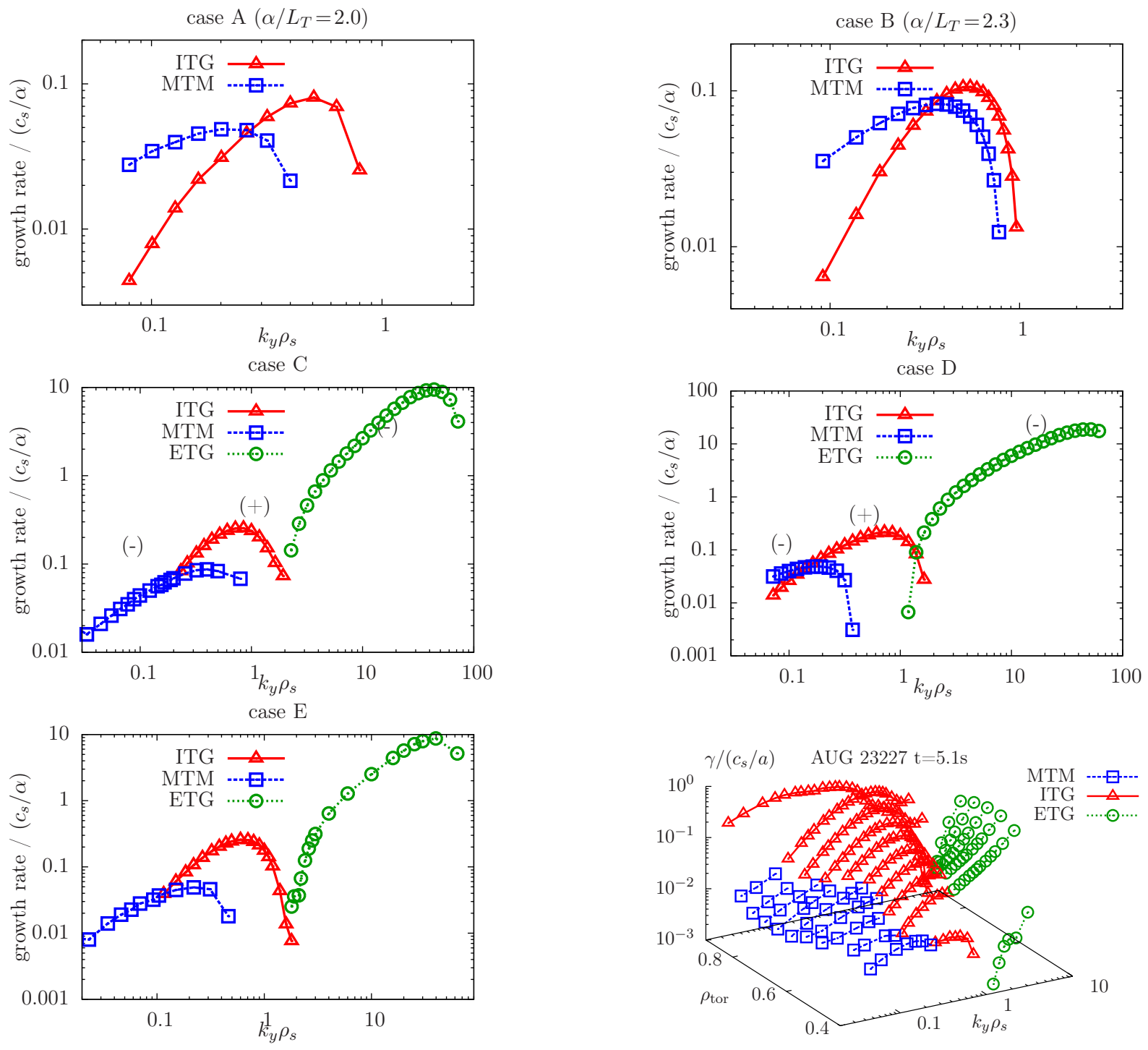

Figure 2: Stability analysis for cases A-E. If modified, the gradient $\alpha / L_{T}$ is specified in the title. Modes propagating in electron drift direction are marked as blue squares (MTM) and green dots (ETG/TEM), while modes propagating in ion drift direction (ITG) are marked by red triangles. For the discharge of case E a range of radial positions is displayed. Microtearing modes co-exist with ITG modes over a wide range in the radial position.

by increased $\alpha / L_{n}$ and $\alpha / L_{T e}$. Importantly, the nominal parameters are rather far from the onset of TEM and KBM instability, but ITG and MTM are well above their thresholds. For AUG\#23227 (from which case $\mathrm{E}$ was selected), we additionally vary the radial position $\rho_{\text {tor }}$ in Fig. 2 to show that the MTM/ITG coexistence spreads over a wide range in the outer core, which is typical for all considered discharges. In cases C-E, ETG/TEM becomes unstable at larger $k_{y}$ wavenumbers, but we will focus on ion-scales $k_{y} \rho_{s} \lesssim 1$ in the following.

\section{B. The impact of $\beta$}

The impact of magnetic fluctuations on microinstabilities is determined by varying $\beta$, as depicted in Fig. 3 for selected wavenumbers. The observed features are quite similar between the cases, although the importance of electromagnetic effects at the experimental operation point can substantially vary. As expected, the ITG mode is stabilized with increased $\beta$ and a KBM gets destabilized above a certain threshold value $\beta_{\text {crit,KBM. }}$. We determine this threshold value from a fit to the growth rates of the KBM branch and minimize over $k_{y}$. The result is summarized in Table. III. The MHD estimate $\beta_{\text {crit,mhd }}=$ $0.6 \hat{s} /\left[q^{2} \sum_{s}\left(p_{0 s} / p_{0 e}\right)\left(R / L_{n s}+R / L_{T s}\right)\right]$ for circu- 

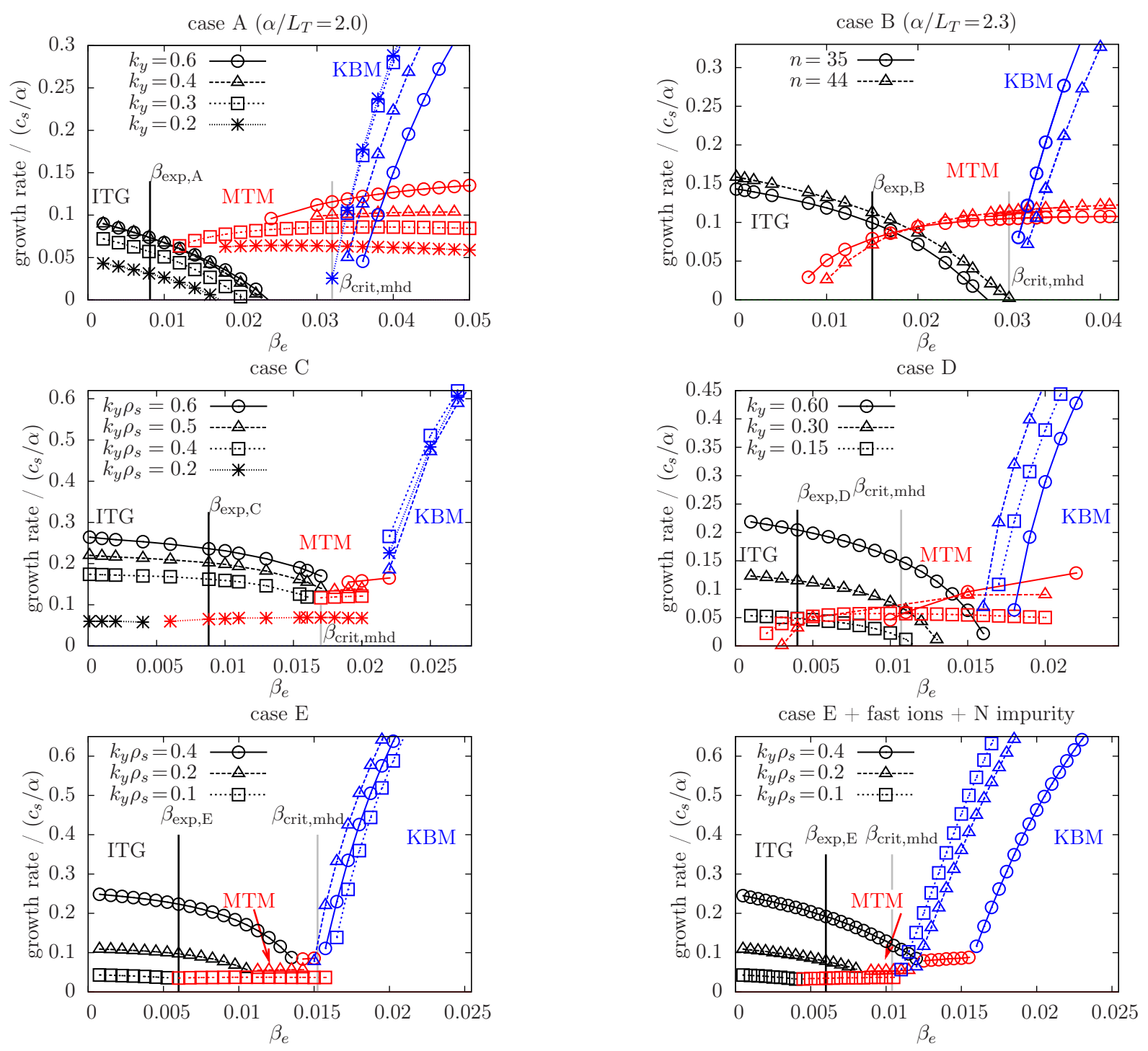

Figure 3: Typical results of $\beta$ variations for various wavenumbers $k_{y}: \beta / \beta_{\text {crit,KBM }}$ is $20 \%, 50 \%, 40 \%, 15 \%$ and $40 \%$ in cases A,B,C,D and E, respectively. In all selected cases, ITG modes (black symbols) are stabilized by magnetic fluctuations, but KBMs (blue symbols) are stable for nominal $\beta$. The MTMs (red symbols) are unstable in all cases, but they are most pronounced in case B.

lar geometry is also given as a reference.

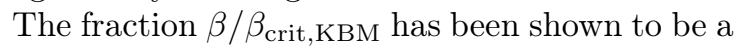
useful parameter to quantify the degree of electromagnetic stabilization, even in the nonlinear case $[44,56]$. Taking this measure, case B is most electromagnetic, with $\beta / \beta_{\text {crit,KBM }}$ around $50 \%$ (depending on the temperature gradient). In case $\mathrm{E}$, the concentration of fast NBI ions is relatively high: we have $n_{f} / n_{e}=0.0276, T_{f} / T_{e}=9.78$, $\alpha / L_{n f}=6.34$ and $\alpha / L_{T f}=0.892$. Including them as a dynamic species (and adding a $Z_{\text {eff }}=1.5$ nitrogen impurity species) is shown to lower the KBM threshold and thus enhance ITG stabilization.

In contrast to the widely-studied Cyclone-Basecase (CBC) [12], TEMs are stable. Instead,
MTMs are present here, which are strongly destabilized by increased $\beta_{e}$, above some linear critical $\beta_{\text {crit } M T M} \lesssim 0.01$. Again, the strongest MTM contribution is found in case B.

\section{Critical gradients}

Strong ITG turbulent transport occurs above a certain gradient threshold $[57,58]$, which is consistent with the experimental finding of stiff profiles. It is thus useful to determine the gradient threshold $\alpha / L_{T, \text { crit }}$ for our cases, which usually depends on the wavenumber. Here, we take the minimum between $0.1<k_{y} \rho_{s}<0.7$. Of special interest are cases $\mathrm{A}$ and $\mathrm{B}$, whose parameters are quite similar, 


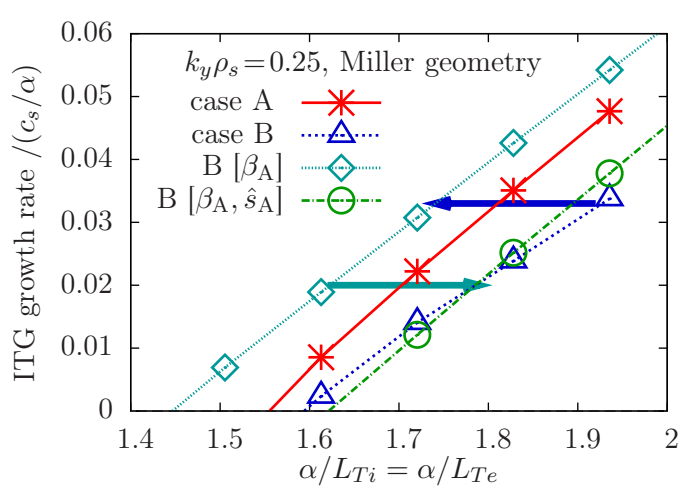

Figure 4: The critical values of $\alpha / L_{T i}$ for the ITG instability is determined for cases $\mathrm{A}$ and $\mathrm{B}$ at $k_{y}=$ 0.25. Employing miller geometry, a $10 \%$ up-shift of $\alpha / L_{T \text { crit }}$ due to $\beta_{A}<\beta_{B}$ is shown to be balanced by a shear decrease $\hat{s}_{A}>\hat{s}_{B}$.

\begin{tabular}{|c|c|c|c|c|c|}
\hline case & $\alpha / L_{T i}$ & $\alpha / L_{T e}$ & $\beta_{\text {crit,mhd }}$ & $\beta_{\text {crit,KBM }}$ & $\beta_{e} / \beta_{\text {crit,KBM }}$ \\
\hline \hline A & 2.0 & 2.0 & 0.0306 & 0.0313 & 0.26 \\
\hline B & 2.3 & 2.3 & 0.0300 & 0.03 & 0.5 \\
\hline C & 2.0 & 2.0 & 0.0170 & 0.0205 & 0.42 \\
\hline D & 2 & 3 & 0.0107 & 0.0161 & 0.25 \\
\hline E & 2.61 & 1.73 & 0.0152 & 0.017 & 0.35 \\
\hline E+fast & 2.61 & 1.73 & 0.0104 & 0.0105 & 0.057 \\
\hline
\end{tabular}

Table III: Beta thresholds for the onset of KBM. A slight temperature gradient modification is introduced for cases A and B. In case E, the KBM threshold is lowered by including fast ions.

except the $\beta$ increase by a factor of two. Due to $\beta$ stabilization, we expect $\left(\alpha / L_{T}\right)_{\text {crit }}$ to be larger in case B. However, the thresholds are almost identical and appear at $k_{y} \rho_{s}=0.25\left(n_{\text {tor }}=35\right)$ in both cases. Fitting Miller geometry parameters to the $\rho_{\text {tor }}=0.5$ flux surface, allows us to show in Fig. 4 that in this case, $\beta$-stabilization is balanced by a slight change in geometry (mainly magnetic shear). Note that growth rates from miller and numerical geometry do not exactly coincide, which indicates the importance of higher order geometry corrections, like up-down asymmetry.

Table IV shows that the critical gradients (as well as the corresponding wavenumber) can be quite different between the five cases. The reason is seen in the dependence on parameters like $q, \hat{s}$ and $x_{0}$, for example.

\begin{tabular}{|c|c|c|}
\hline case & ITG $\alpha / L_{T i, \text { crit }}\left(k_{y} \rho_{s}\right)$ & MTM $\alpha / L_{T e, \text { crit }}\left(k_{y} \rho_{s}\right)$ \\
\hline \hline A & $1.6(0.35)$ & $0.8(<0.1)$ \\
\hline B & $1.6(0.35)$ & $0.8(<0.1))$ \\
\hline C & $0.6(0.7)$ & $<0.6(<0.1)$ \\
\hline D & $0.8(0.4)$ & $<0.7(<0.1)$ \\
\hline E & $\sim 0.86(0.6)$ & $0.6(0.1)$ \\
\hline E+fast & $\sim 0.85(0.6)$ & $0.6(0.1)$ \\
\hline
\end{tabular}

Table IV: Critical gradient for ITG and MTM in cases A-F, minimized over wavenumber (given in brackets) between $0.1<k_{y} \rho_{s}<0.7$.

\section{NONLINEAR GENE SIMULATIONS AND COMPARISON TO THE EXPERIMENT}

\section{A. Relevant nonlinear physics}

In this section, a first-principles description of turbulent transport is provided by performing nonlinear GENE simulations for the selected parameter sets. We begin with a discussion of the relevant basic physics mechanisms. Later on, we compare turbulent energy fluxes to experimental results. The question, whether the coexistence of MTM and ITG modes carries over to the nonlinear regime is addressed first. In this context, case B is most interesting, because MTM and ITG growth rates are comparable. One could thus expect from quasilinear theory that the heat fluxes related to MTM and ITG turbulence are of similar magnitude as well.

A first nonlinear simulation (B.1) (for which we neglect $\gamma_{E}$ shearing), almost no signature of linearly unstable microtearing modes is observed, though. For illustration, Fig. 5 depicts transport spectra and turbulent time traces. The magnetic component $Q_{e}^{\mathrm{em}}$ contributes less than $10 \%$ of the total heat flux. In the previously studied CBC the magnetic contribution is about $20 \%$ for the same $\beta / \beta_{\text {crit,KBM }}[12]$. There, $Q_{e}^{\mathrm{em}}$ has been shown to decompose into an inward quasilinear (ballooning parity) and an outward tearing parity part, which is related to nonlinearly excited subdominant MTMs [38, 39]. However, the $k_{y}$ spectrum shown in Fig. 5 appears to be inverted with respect to the CBC. It is yet to be clarified, whether linearly unstable MTMs are responsible for that feature. Also for ITER baseline H-mode parameters, MTMs have been found linearly unstable, while the integrated magnetic flutter transport is insignificant in corresponding nonlinear gyrokinetic simulations, [59]. These cases thus differ from previous reports on microtearing turbulence, which - for slightly different parameter setupshas been shown to yield relevant magnetic flux lev- 

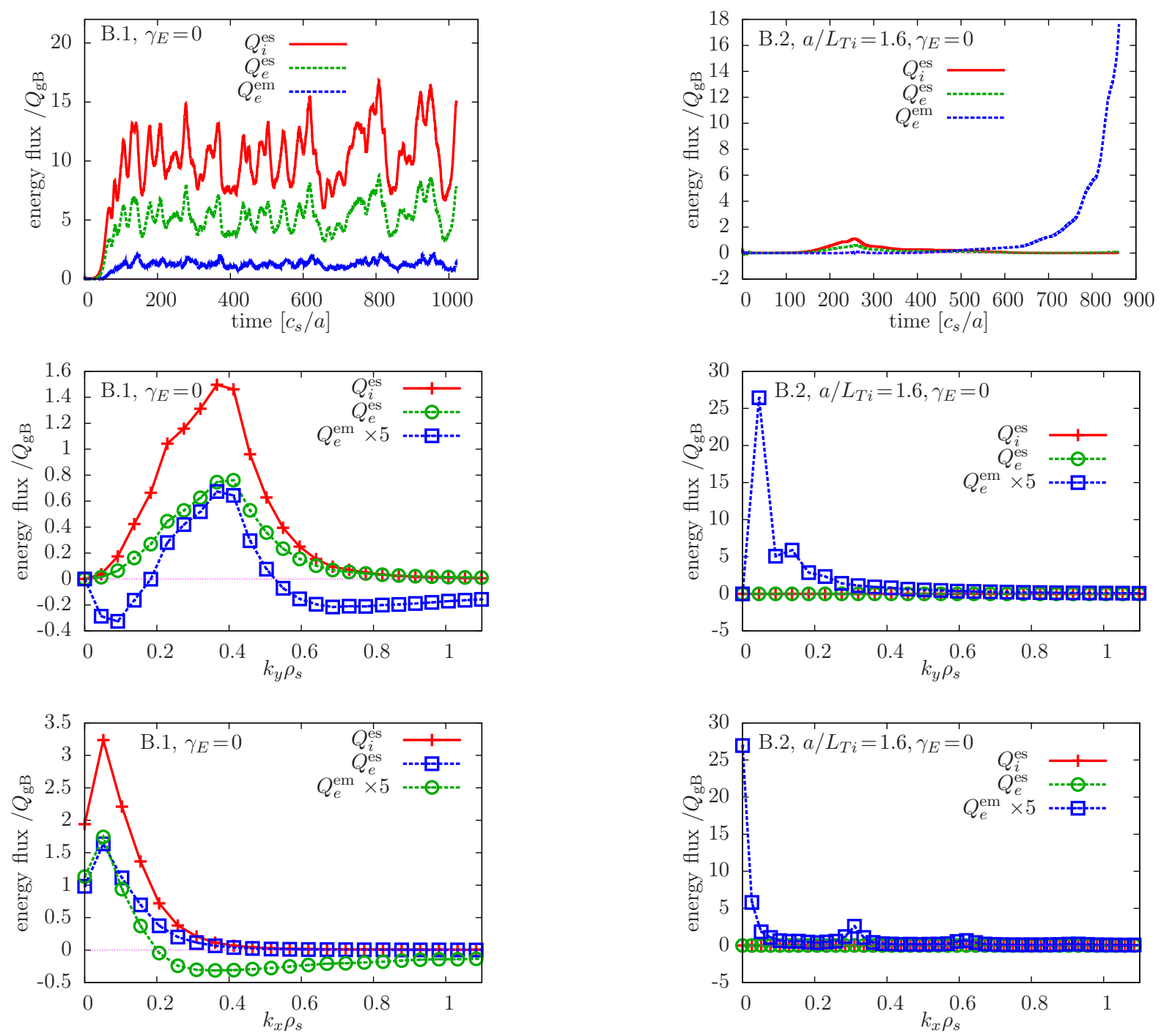

Figure 5: Nonlinear simulation results for case B) B.1 has $\alpha / L_{T i}=\alpha / L_{T e}=2.3$. (time average for $100<\hat{t}<1050$ ). B.2 has $\alpha / L_{T i}=1.6$, below the nonlinear ITG threshold, which lets the MTM develop unphysical magnetic streamers of $k_{y}=k_{y, \min }$ and $k_{x}=0(800<\hat{t}<860)$.

els in standard tokamaks [22, 27]. For the spherical tokamak NSTX, where plasma parameters deviate more strongly, certain transport scalings are found to be consistent with MTM turbulence [23, 24].

Is microtearing activity thus suppressed by the presence of an ITG turbulence background? To address this question, a test simulation (B.2) has been set up at $\alpha / L_{T i}=1.6$ (close to the linear critical gradient), where ITG turbulence is expected to be extremely weak. In this simulation, we observe microtearing modes (Fig. 5), whose amplitudes peak at the largest wavelength (box-size) in $y$ and forms magnetic streamers reaching across the radial domain. This artifact is not removed by increasing the domain to up to $l_{x}=250 \rho_{s}$. Since even larger flux-tubes are unrealistic, no statement on MTM turbulence in the hypothetical scenario of a flattened ion temperature can be made at present. A global approach that considers profile variations might be successful, but this has not yet been attempted. However, we can already conclude that the presence of an ITG turbulent background removes radially extended MTM streamers for case B. As we will see later on in this section, including equilibrium flow shear has a similar effect. Thus, there has to be a mechanism that either prevents microtearing modes from growing or saturates them at a low amplitude.

One possible reason could be the shearing effect of zonal flows or zonal fields, which are generated by ITG turbulence. To quantify this, a tertiary stability analysis is performed following 
the procedure described in Ref. [60]. To that aim, we evaluate the modification of the electron temperature gradient fluctuations (performing a $y$ and $z$ average), as well as the shear of zonal flow $\left(\mathrm{d} \phi_{\text {zonal }} / \mathrm{d} x\right)$ and zonal field $\left(\mathrm{d} A_{\| \text {zonal }} / \mathrm{d} x\right)$, as depicted in Fig. 6. The effect of zonal flow shearing on the linear instabilities is modelled by adding (in normalized units) a term of the form $v_{E 0}\left(i k_{y}\right) g_{1 j}$ to the gyrokinetic equation for species $j$, where $g_{1}=f_{1}-v_{\|} f_{M} A_{1 \|} / T_{0}$. For the effective drift $v_{E 0}=-i k_{x 0} \phi_{0}$, the potential $\phi_{0}$ and the wavenumber $k_{x, 0}$ are chosen to represent the zonal flow generated in the full nonlinear simulation. While ITG growth rates tend to be reduced by such a term - in accordance with predator-prey modelsthe MTM growth rate is even slightly enhanced. The same applies to a modeled zonal field shearing that is implemented in a similar fashion, but using $v_{B 0}=i k_{x 0} v_{\|} v_{T, j} A_{\| 0}$ as a drift. Typical values for $k_{x 0}$ and $A_{\| 0}$ are again extracted from the full turbulence data. Corrugations of the electron temperature profile are expected to reduce the linear drive, since $\alpha / L_{T e}$ is the most relevant parameter for MTM instability. Maximal effective reduction is indeed found in close proximity to rational flux surfaces (see also Refs. [61]), where the MTM drive is located. However, $\alpha / L_{T e}$ is only lowered by about $25 \%$, which is not sufficient to stabilize the mode (compare Fig. 4 and Table IV). Apparently, such simple models fail to explain MTM suppression in an ITG turbulent background, leaving this question open for future research.
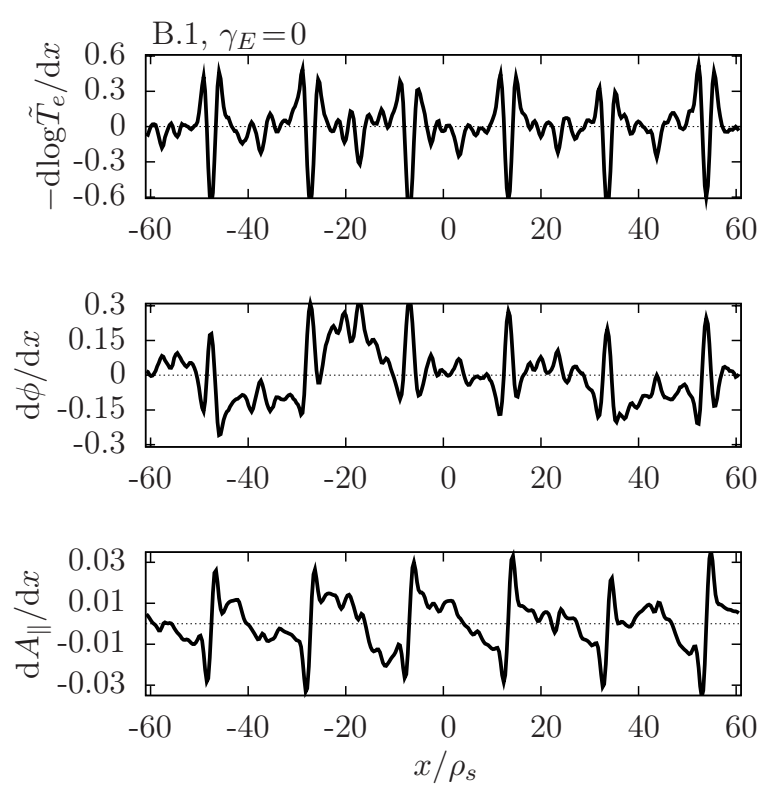

Figure 6: The electron temperature profile corrugations for case B without external $\left(\gamma_{E}\right)$ shear. Additionally, the zonal flow and zonal field shearing is depicted.
In all following simulations, we add background flow shear, the rate $\gamma_{E}$ being determined from toroidal rotation measurements. Simulation B.3 uses $\alpha / L_{T i}=2.3$ and B.4 has $\alpha / L_{T i}=1.6$, while the electron gradient is $\alpha / L_{T e}=2.3$ in both cases. Time-averaged turbulence spectra and time traces of spatially averaged fluxes are shown in Fig. 7. In the ITG dominated case, B.3, equilibrium flow shear reduces ITG transport by about a factor of four. The ratio $Q^{\mathrm{em}} / Q^{\mathrm{es}}$ is reduced as well. Interestingly, the shape of the $Q_{e}^{\mathrm{em}}$ spectrum changes qualitatively towards the quasilinear expectation of an inward directed fraction of $Q^{\text {es }}$. A tiny low $k_{y}$ peak of $Q_{e}^{\mathrm{em}}$ might be an indication for linearly unstable MTMs, which, however, remains at small amplitude throughout the simulation over $800 \alpha / c_{s}$ time units. Simulation B. 4 shows that in the absence of ITG turbulence, equilibrium flow shear lets MTM saturate at a low turbulence level. The total heat flux in the pure MTM simulation B.4 is about an order of magnitude below the experimental result, while B.3 yields a somewhat larger total flux.

In conclusion, our gyrokinetic simulation results indicate that turbulent transport is mainly driven by ITG modes in the considered cases. Even though MTMs are linearly unstable at lower or comparable $k_{y}$, they do not cause significant transport. Since $\beta$ is below $50 \%$ of the KBM threshold, KBM turbulence does not play a role either.

\section{B. Comparison of cases A and B to experimental power balance analyses}

In the following, we study the impact of $\beta$ on the ITG turbulence level by comparing cases A and B and relate the simulation results to experimental findings. It is well known that transport levels can be quite sensitive to the temperature gradient, so that a variation around the experimentally determined nominal value proves helpful.

Figure 8 depicts the total turbulent heat flux for cases $\mathrm{A}$ and $\mathrm{B}$ as a function of $\alpha / L_{T}$, as well as the linear gradient thresholds. Electron and ion gradients are changed simultaneously. Two main observations can be made. First, the turbulent heat transport is extremely sensitive to $\alpha / L_{T}$ in the simulation reflected in the experiments by the fact that $\alpha / L_{T}$ is expected to be close to its critical value. Second, the gradient threshold for the onset of strong transport experiences a substantial nonlinear up-shift [57]. Since case B mainly differs from case $\mathrm{A}$ by a doubled value of $\beta$, we conclude that this nonlinear up-shift increases with $\beta$. Indeed, replacing only $\beta_{A} \rightarrow \beta_{B}$ in case $\mathrm{A}$, substantially reduces $Q^{A}$, down to case $\mathrm{B}$ levels (green diamonds in Fig. 8). 

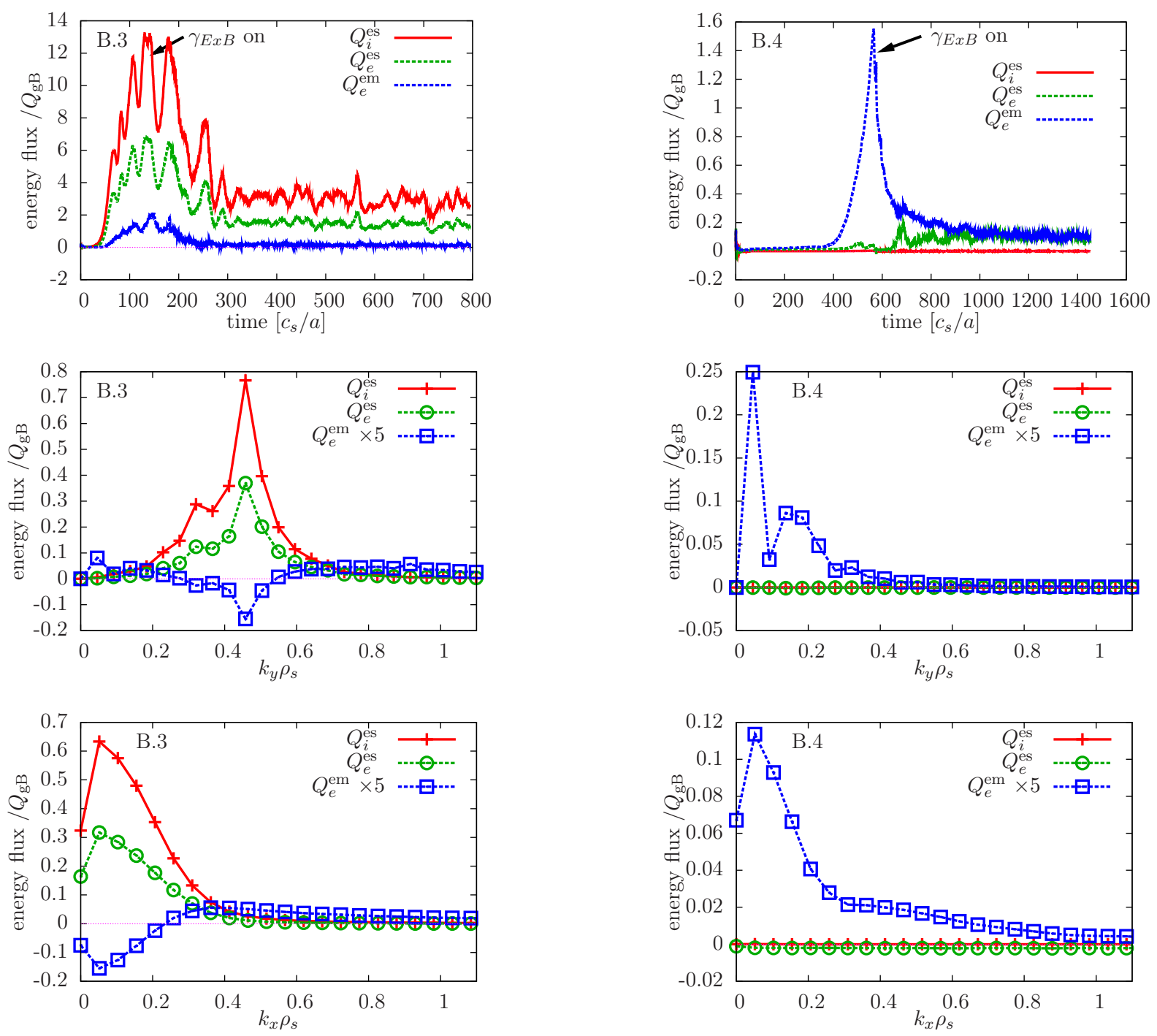

Figure 7: Nonlinear simulation results for case B.3 and B.4.

Overall, these results qualitatively agree with previous discoveries on electromagnetic ITG turbulence in simplified setups $[11,12,56]$. Also recent gyrokinetic simulation work for JET parameters (with $\beta / \beta_{\text {crit }, K B M}$ being significantly larger) show similar trends $[43,44]$. There, electromagnetic stabilization leads to stiffness reduction in cases of low magnetic shear at $\rho_{\text {tor }}=0.33$, while in the outer core $\left(\rho_{\text {tor }}=0.65\right)$, the stabilization due to $\gamma_{E}$ is much more pronounced. The present ASDEX Upgrade $\beta$ scan, consisting of cases A and $\mathrm{B}$ seems to lie in between: both electromagnetic effects and flow shear reduce ITG transport. Stiffness is not reduced due to higher $\beta$, however.

For comparison to the experiment, in Fig. 8, the TRANSP power balance results $Q^{\exp }$ are marked at the measured values of $\alpha / L_{T}$. We define the energy flux as $Q^{\exp }=P / V^{\prime}$, where $P$ is the to- tal power transported through the $\rho_{\text {tor }}=0.5$ flux surface of the area $V^{\prime}$. The uncertainties are assumed as \pm 0.5 for $\alpha / L_{T}$ (compare Fig. 1) and $\pm 20 \%$ for the experimental flux. The gyro-Bohm

\begin{tabular}{|c|c|c|c|c|}
\hline case & $P / \mathrm{MW}$ & $Q_{\mathrm{gB}} /\left(\mathrm{MW} / \mathrm{m}^{2}\right)$ & $P /\left(V^{\prime} Q_{\mathrm{gB}}\right)$ & $V^{\prime}$ \\
\hline $\mathrm{A}$ & 3.76 & 0.064 & 2.67 & 22 \\
\hline $\mathrm{B}$ & 6.0 & 0.199 & 1.37 & 22 \\
\hline
\end{tabular}

Table V: Power $P$ through the $\rho_{\text {tor }}=0.5$ surface (from TRANSP), gyro-Bohm unit and flux-surface area $V^{\prime}$ are given for cases $\mathrm{A}$ and $\mathrm{B}$.

flux $Q_{\mathrm{gB}}=p_{0 e} c_{s} \rho^{\star 2}$, which is used for normalization, has a strong dependence on temperature $Q_{\mathrm{gB}} \sim T^{5 / 2}$. As Table $\mathrm{V}$ shows, $P / V^{\prime}$ increases less rapidly from case A to case B than $Q_{\mathrm{gB}}$ does. This is the reason for the power-balance result 


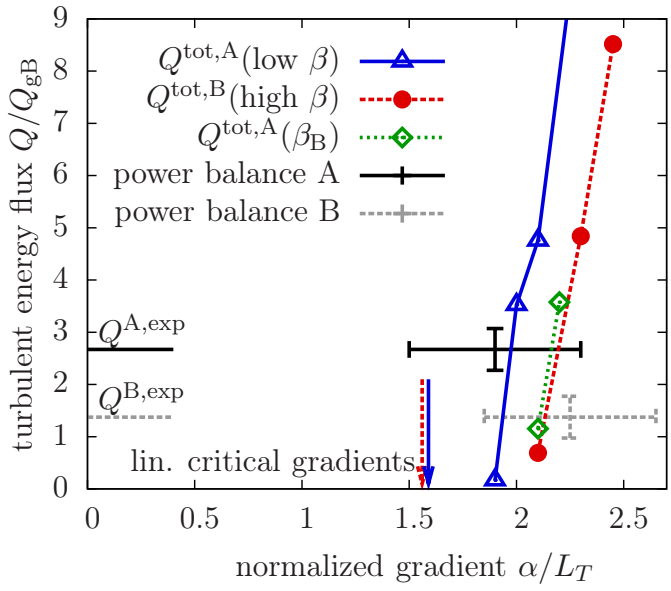

Figure 8: Dependence of the total heat flux on the temperature gradient for cases A and B. A nonlinear up-shift of the ITG threshold is visible, which increases with $\beta$. The fluxes from TRANSP power balance as well as the measured gradients are shown for comparison. A trend of higher $\alpha / L_{T}$ at higher $\beta$ can be identified in experiment and simulation, but the experimental uncertainties do not allow definite conclusions.

$Q^{\mathrm{B}, \exp }$ to appear smaller than $Q^{\mathrm{A} \text {,exp }}$ in Fig. 8 and indicates that gyro-Bohm scaling does not strictly apply at the considered plasma radius. Potential reasons being manifold, one interpretation is that gyro-Bohm scaling is too pessimistic and that beta-induced transport reduction plays a role in the core. Moreover, the observed effect on global confinement $\tau_{\mathrm{E}, \mathrm{th}} B_{t}$ (see Table I) is weak in the present discharges, which likely relates to edge physics.

For $\rho_{\text {tor }}=0.5$, Fig. 8 shows that experimental and modelling values of $\alpha / L_{T}$ are consistent within the experimental error bars. Due to the high stiffness in the simulation data and due to the relatively high uncertainty of the experimental temperature gradient, no definite conclusions regarding the heat fluxes can be drawn at this point. Interestingly, electrostatic simulations yield total heat flux levels that are only slightly larger than the ones of the low beta case. One should, however, not be mislead in concluding that electrostatic physics suffices to describe tokamak transport. In the discharges analyzed here, electromagnetic effects significantly impact on the turbulence level when $\beta / \beta_{\text {crit,KBM }}$ becomes of the order of $40 \%$ or larger. Importantly, the trend of higher $\alpha / L_{T}$ at higher $\beta$ is visible in the temperature data (Fig. 1) in the outer core, as well as in gyrokinetic simulations at $\rho_{\text {tor }}=0.5$. This is clear evidence for the dominant role of ITG turbulence, and the importance of stabilizing $\beta$-effects, which will increase at higher $\beta$.

The analysis of more outward radial positions is expected to further consolidate these arguments, but this is left for future work. Due to the extremely high sensitivity to the gradients, it will be helpful to repeat the experiments with even enhanced measurement accuracy. Improved diagnostics are already installed at ASDEX Upgrade.

\section{Discussion of supporting cases C,D and E}

After discussing the beta variation cases $\mathrm{A}$ and $\mathrm{B}$, we now turn to a brief discussion of the nonlinear results for the supporting cases $\mathrm{C}-\mathrm{E}$, with a focus on ion-scale turbulence only. Linear results indicate that in these cases, ETG and/or TEM modes may contribute to turbulent transport at higher wavenumbers. This would be clarified by electron-ion multiscale simulations [62-64], which are not attempted here, however.

Case $\mathrm{C}$ is taken from the same discharge as case $\mathrm{B}$, but at $\rho_{\text {tor }}=0.75$, thus representing the first step in further analysis of the beta scaling discharges. Here, linear results (Fig. 3) show only a moderate reduction of the ITG growth rate compared to the electrostatic result, even though $\beta / \beta_{\text {crit }}$ is comparable to case B. Two species turbulence simulations for case $\mathrm{C}$ require a reduction of the ion temperature gradient from the experimental value $\alpha / L_{T i}=3$ to $\alpha / L_{T i} \sim 2$ to match the fluxes of a TRANSP run. This apparent inconsistency between simulation and experiment needs to be further investigated to draw definite conclusions. The comparison with a low-beta case requires improved measurements at the outward radial positions. Fast ions may play a stabilizing role, as discussed in Refs. [43, 65]. Indeed, TRANSP runs indicate a peak of the fast ion density around $\rho_{\text {tor }}=0.7$, attributed to the fact that the neutral beam heating system has a limited penetration depth at high plasma density. It can already be concluded at the present stage that MTMs are nonlinearly suppressed in this case as well.

Also in case D, no nonlinear MTM activity is seen and ITG turbulence dominates instead. Transport levels are quite insensitive to (the experimental value of) equilibrium flow shear. A nonlinear gradient scan has not been performed, but gyrokinetic predictions for heat fluxes are quite close to the expected level.

Case E has originally been selected for its high content of fast ions from neutral beam heating. Linear results of Fig. 3 show ITG stabilization due to these fast ions. For the nonlinear case, we perform two-species simulations as a first step and we find ITG to dominate over MTM turbulence, which is consistent with cases A-D. Matching experimental and gyrokinetic power fluxes has not succeeded yet in this simplified setup. Nonlinear multi-ion 
studies including impurities and fast ions are subject to future research.

\section{CONCLUSIONS}

We have performed a gyrokinetic study on a set of four ASDEX Upgrade H-Mode discharges, with a focus on unraveling the role of miscellaneous electromagnetic effects. Two of those discharges (cases A and B) are extremely useful in this context. They have been designed to solely vary $\beta$ and leave other dimensionless parameters constant. Linear simulations at $\rho_{\text {tor }}=0.5$ showed that these experimentally relevant cases are subject to robust ITG and MTM instability, but they are well below the $\mathrm{KBM}$ threshold $\left(\beta / \beta_{\text {crit, KBM }} \leq 50 \%\right)$. MTM growth rates can become comparable to ITG growth rates, depending on $\beta$ and gradients, and their wavenumber spectra can widely overlap. Nonlinear simulations revealed, however, that ITG transport dominates by far. Thus, without claim of generality, the (dynamical) electromagnetic stabilization of ITG turbulence is the most relevant $\beta$-effect in these experimentally relevant cases.

Linear and nonlinear simulations for three further parameter sets C, D, and E (at more outward radial positions up to $\left.\rho_{\text {tor }}=0.75\right)$ show similar basic properties and thus support this statement. In the latter three cases, ETG and/or TEM modes may additionally contribute to turbulent transport at higher wavenumbers or even back-react to ion scales. Clarifying multiscale simulations have not been attempted yet.

Furthermore, it remains an open question, why microtearing modes are suppressed, while they can lead to substantial electron heat flux for different parameters. Simple tests showed that profile corrugations likely are not responsible.

Comprehensive nonlinear simulations, including gradient scans, have been performed for cases A and B. In qualitative agreement with simplified setups, a nonlinear up-shift of the critical gradient is observed, which increases with $\beta$. Importantly, the trend of increased logarithmic temperature gradient is visible both in experiment and modelling. Due to high stiffness in the simulation data, this trend is within the experimental uncertainty of the gradients. Nonetheless, electromagnetic stabilization is found to be essential, so that an improvement of core confinement with increased $\beta$ is suggested by the simulation results. This contradicts the widely used IPB98 $(y, 2)$ confinement scaling of
H-mode plasmas that involves a rather strong $\beta$ degradation $\tau_{E} B_{0} \propto \beta^{-0.9}$.

The present pair of ASDEX Upgrade discharges show a weak increase of the normalized global confinement time $\Omega_{i} \tau_{E}$ between the low and the high beta cases. Obviously, a conclusive prediction of global plasma confinement can only be made, if measurement precision-particularly in the gradients - is further increased, and more outward radial positions, as well as the plasma edge, are considered.

However, already now, we are confident that the main physics ingredients are robust and that electromagnetic stabilization of ITG turbulence plays an important role for core transport in highperformance tokamak plasmas. This should be considered for extrapolations to future machines and underlines the potential need for refined dimensionless parameter scaling laws.

\section{Acknowledgements}

The authors would like to thank J. Abiteboul, A. Bañon Navarro, F. J. Casson, T. Görler, D. R. Hatch, K. Lackner, M. J. Pueschel and D. Told for valuable discussions.

This work has been carried out within the framework of the EUROfusion Consortium and has received funding from the Euratom research and training programme 2014-2018 under grant agreement No 633053. The views and opinions expressed herein do not necessarily reflect those of the European Commission.

The research leading to these results has received funding from the European Research Council under the European Unions Seventh Framework Programme (FP7/2007-2013)/ERC Grant Agreement No. 277870 .

The numerical results presented in this work were carried out using the HELIOS supercomputer system at the Computational Simulation Centre of International Fusion Energy Research Centre (IFERC-CSC), Aomori, Japan, under the Broader Approach collaboration between Euratom and Japan, implemented by Fusion for Energy and JAEA and using the resources of the RZG computing center, Garching, Germany.

\section{References}

[1] F. Wagner, G. Becker, K. Behringer, D. Campbell, A. Eberhagen, W. Engelhardt, G. Fussmann,
O. Gehre, J. Gernhardt, G. v. Gierke, et al., Phys. 
Rev. Lett. 49, 1408 (1982).

[2] D. C. McDonald, J. G. Cordey, C. C. Petty, M. Beurskens, R. Budny, I. Coffey, M. de Baar, C. Giroud, E. Joffrin, PLomas, et al., Plasma Phys. Control. Fusion 46, A215 (2004).

[3] C. C. Petty, T. C. Luce, D. C. McDonald, J. Mandrekas, M. R. Wade, J. Candy, J. G. Cordey, V. Drozdov, T. E. Evans, J. R. Ferron, et al., Phys. Plasmas 11, 2514 (2004).

[4] H. Urano, T. Takizuka, H. Takenaga, N. Oyama, Y. Miura, and Y. Kamada, Nucl. Fusion 46, 781 (2006).

[5] L. Vermare, F. Ryter, C. Angioni, A. G. Peeters, J. Stober, R. Bilato, L. D. Horton, B. Kurzan, C. F. Maggi, H. Meister, et al., Nucl. Fusion 47, 490 (2007).

[6] D. C. McDonald, L. Laborde, J. C. DeBoo, F. Ryter, M. Brix, C. D. Challis, P. de Vries, C. Giroud, J. Hobirk, D. Howell, et al., Plasma Phys. Control. Fusion 50, 124013 (2008).

[7] T. C. Luce, C. C. Petty, and J. G. Cordey, Plasma Phys. Control. Fusion 50, 043001 (2008).

[8] C. C. Petty, Phys. Plasmas 15, 080501 (2008).

[9] I. P. Basis, Nucl. Fusion 39, 2175 (1999).

[10] F. Jenko and W. Dorland, Plasma Phys. Control. Fusion 43, A260000 (2001).

[11] J. Candy, Phys. Plasmas 12, 072307 (2005).

[12] M. J. Pueschel, M. Kammerer, and F. Jenko, Phys. Plasmas 15, 102310 (2008).

[13] M. J. Pueschel and F. Jenko, Phys. Plasmas 17, 062307 (2010).

[14] J. Y. Kim, W. Horton, and J. Q. Dong, Phys. Fluids B 5, 4030 (1993).

[15] E. A. Belli and J. Candy, Phys. Plasmas 17, 112314 (2010).

[16] S. Maeyama, A. Ishizawa, T.-H. Watanabe, M. Nakata, N. Miyato, M. Yagi, and Y. Idomura, Phys. Plasmas 21, 052301 (2014).

[17] R. D. Hazeltine, D. Dobrott, and T. S. Wang, Phys. Fluids 18, 1778 (1975).

[18] J. F. Drake and Y. C. Lee, Phys. Fluids 20, 1341 (1977).

[19] J. F. Drake, N. T. Gladd, C. S. Liu, and C. L. Chang, Phys. Rev. Lett. 44, 994 (1980).

[20] D. A. Dippolito, Y. C. Lee, and J. F. Drake, Phys. Fluids 23, 771 (1980).

[21] K. L. Wong, S. Kaye, D. R. Mikkelsen, J. A. Krommes, K. Hill, R. Bell, and B. Leblanc, Phys. Rev. Lett. 99, 135003 (2007).

[22] H. Doerk, F. Jenko, M. J. Pueschel, and D. R. Hatch, Phys. Rev. Lett. 106, 155003 (2011).

[23] W. Guttenfelder, J. Candy, S. M. Kaye, W. M. Nevins, E. Wang, R. E. Bell, G. W. Hammett, B. P. Leblanc, D. R. Mikkelsen, and H. Yuh, Phys. Rev. Lett. 106, 155004 (2011).

[24] W. Guttenfelder, J. L. Peterson, J. Candy, S. M. Kaye, Y. Ren, R. E. Bell, G. W. Hammett, B. P. LeBlanc, D. R. Mikkelsen, W. M. Nevins, et al., Nucl. Fusion 53, 093022 (2013).

[25] D. J. Applegate, C. M. Roach, J. W. Connor, S. C. Cowley, W. Dorland, R. J. Hastie, and N. Joiner, Plasma Phys. Control. Fusion 49, 1113 (2007).

[26] L. Vermare, C. Angioni, A. Bottino, A. G. Peeters, and ASDEX Upgrade Team, J. Phys. Conference
Series 123, 012040 (2008).

[27] H. Doerk, F. Jenko, T. Görler, D. Told, M. J. Pueschel, and D. R. Hatch, Phys. Plasmas 19, 055907 (pages 12) (2012).

[28] W. Guttenfelder, J. Candy, S. M. Kaye, W. M. Nevins, R. E. Bell, G. W. Hammett, B. P. Leblanc, and H. Yuh, Phys. Plasmas 19, 022506 (2012).

[29] D. Dickinson, C. M. Roach, S. Saarelma, R. Scannell, A. Kirk, and H. R. Wilson, Plasma Phys. Control. Fusion 55, 074006 (2013).

[30] D. Dickinson, C. M. Roach, S. Saarelma, R. Scannell, A. Kirk, and H. R. Wilson, Phys. Rev. Lett. 108, 135002 (2012).

[31] J. Canik, W. Guttenfelder, R. Maingi, T. Osborne, S. Kubota, Y. Ren, R. Bell, H. Kugel, B. LeBlanc, and V. Souhkanovskii, Nuclear Fusion 53, 113016 (2013).

[32] D. Told, F. Jenko, P. Xanthopoulos, L. D. Horton, E. Wolfrum, and ASDEX Upgrade Team, Phys. Plasmas 15, 102306 (2008).

[33] S. Saarelma, M. N. A. Beurskens, D. Dickinson, L. Frassinetti, M. J. Leyland, C. M. Roach, and E.-J. Contributors, Nucl. Fusion 53, 123012 (2013), 1301.2919.

[34] M. J. Pueschel, P. W. Terry, F. Jenko, D. R. Hatch, W. M. Nevins, T. Görler, and D. Told, Phys. Rev. Lett. 110, 155005 (2013).

[35] M. J. Pueschel, P. W. Terry, and D. R. Hatch, Phys. Plasmas 21, 055901 (2014).

[36] W. M. Nevins, E. Wang, and J. Candy, Phys. Rev. Lett. 106, 065003 (2011).

[37] E. Wang, W. M. Nevins, J. Candy, D. Hatch, P. Terry, and W. Guttenfelder, Phys. Plasmas 18, 056111 (2011).

[38] D. R. Hatch, M. J. Pueschel, F. Jenko, W. M. Nevins, P. W. Terry, and H. Doerk, Phys. Rev. Lett. 108, 235002 (2012).

[39] D. R. Hatch, M. J. Pueschel, F. Jenko, W. M. Nevins, P. W. Terry, and H. Doerk, Phys. Plasmas 20, 012307 (2013).

[40] D. Told, F. Jenko, T. Görler, F. J. Casson, E. Fable, and ASDEX Upgrade Team, Phys. Plasmas 20, 122312 (2013).

[41] T. Görler, A. White, D. Told, F. Jenko, C. Holland, and T. L. Rhodes, submitted to Phys. Plasmas (2014).

[42] C. Holland, J. E. Kinsey, J. C. DeBoo, K. H. Burrell, T. C. Luce, S. P. Smith, C. C. Petty, A. E. White, T. L. Rhodes, L. Schmitz, et al., Nucl. Fusion 53, 083027 (2013).

[43] J. Citrin, F. Jenko, P. Mantica, D. Told, C. Bourdelle, J. Garcia, J. W. Haverkort, G. M. D. Hogeweij, T. Johnson, and M. J. Pueschel, Phys. Rev. Lett. 111, 155001 (2013).

[44] J. Citrin, F. Jenko, P. Mantica, D. Told, C. Bourdelle, R. Dumont, J. Garcia, J. W. Haverkort, G. M. D. Hogeweij, T. Johnson, et al., Nuclear Fusion 54, 023008 (2014).

[45] F. Jenko, W. Dorland, M. Kotschenreuther, and B. N. Rogers, Phys. Plasmas 7, 1904 (2000).

[46] F. Merz, Ph.D. thesis, Universität Münster (2009).

[47] H. Doerk, Ph.D. thesis, Universität Ulm (2012).

[48] T. Görler, X. Lapillonne, S. Brunner, T. Dannert, F. Jenko, F. Merz, and D. Told, J. Comput. Phys. 
230, 7053 (2011).

[49] A. Bañón Navarro, B. Teaca, F. Jenko, G. W. Hammett, and T. Happel, Phys. Plasmas 21, 032304 (2014).

[50] M. A. Beer, S. C. Cowley, and G. W. Hammett, Phys. Plasmas 2, 2687 (1995).

[51] P. J. Mc Carthy, Physics of Plasmas (1994present) 6, 3554 (1999).

[52] A. Pankin, D. McCune, R. Andre, G. Bateman, and A. Kritz, Comput. Phys. Commun. 159, 157 (2004).

[53] E. Wolfrum, M. Bernert, J. E. Boom, A. Burckhart, I. G. J. Classen, G. D. Conway, T. Eich, R. Fischer, A. Gude, A. Herrmann, et al., Plasma Phys. Control. Fusion 53, 085026 (2011).

[54] J. Boom, E. Wolfrum, I. Classen, P. de Vries, M. Maraschek, W. Suttrop, C. P. von Thun, A. Donné, B. Tobias, C. Domier, et al., Nucl. Fusion 52, 114004 (2012).

[55] A. Sips, G. Tardini, C. Forest, O. Gruber, P. M. Carthy, A. Gude, L. Horton, V. Igochine, O. Kardaun, C. Maggi, et al., Nucl. Fusion 47, 1485 (2007).

[56] M. J. Pueschel, T. Dannert, and F. Jenko, Comput. Phys. Commun. 181, 1428 (2010).

[57] A. M. Dimits, G. Bateman, M. A. Beer, B. I. Co- hen, W. Dorland, G. W. Hammett, C. Kim, J. E. Kinsey, M. Kotschenreuther, A. H. Kritz, et al., Phys. Plasmas 7, 969 (2000).

[58] E. Asp, J. Weiland, X. Garbet, V. Parail, P. Strand, and t. JET EFDA contributors, Plasma Phys. Control. Fusion 49, 1221 (2007).

[59] F. J. Casson, E. Poli, C. Angioni, R. Buchholz, and A. G. Peeters, Nucl. Fusion 55, 012002 (2015).

[60] M. J. Pueschel, T. Görler, F. Jenko, D. R. Hatch, and A. J. Cianciara, Phys. Plasmas 20, 102308 (2013).

[61] J. Dominski, S. Brunner, S. K. Aghdam, T. Görler, F. Jenko, and D. Told, J Phys Conf Ser 401, 012006 (2012).

[62] T. Görler and F. Jenko, Phys. Rev. Lett. 100, 185002 (2008).

[63] S. Maeyama, Contribution to IAEA fusion energy conference 2014, to be published.

[64] N. T. Howard, A. E. White, M. Greenwald, C. Holland, and J. Candy, Phys. Plasmas 21, 032308 (2014).

[65] C. Holland, C. C. Petty, L. Schmitz, K. H. Burrell, G. R. McKee, T. L. Rhodes, and J. Candy, Nucl. Fusion 52, 114007 (2012). 